\title{
Peritoneal Disorder
}

National Cancer Institute

\section{Source}

National Cancer Institute. Peritoneal Disorder. NCI Thesaurus. Code C26848.

A non-neoplastic or neoplastic disorder that affects the peritoneum. Representative examples of non-neoplastic disorders include peritonitis and panniculitis. Representative examples of neoplastic disorders include adenomatoid tumor, primary peritoneal carcinoma, metastatic carcinoma to the peritoneum, and malignant mesothelioma. 